\title{
Cryogenic Stress-Driven Grain Growth Observed via Microcompression with in situ Electron Backscatter Diffraction
}

\author{
D. FRAZER, ${ }^{1,2}$ J.L. BAIR, ${ }^{3,4}$ E.R. HOMER, ${ }^{3,6}$ and P. HOSEMANN ${ }^{1,5,7}$ \\ 1.-Nuclear Engineering Department, University of California-Berkeley, Berkeley, CA, USA. \\ 2.-Material Science and Technology Division, Los Alamos National Laboratory, Los Alamos, NM, USA. \\ 3.-Department of Mechanical Engineering, Brigham Young University, Provo, UT, USA. \\ 4.-Predictive Chemical and Nuclear Simulations, Pacific Northwest National Laboratory, \\ Richland, WA, USA. 5.-Material Science Division, Lawrence Berkeley National Laboratory, \\ Berkeley, CA, USA. 6.—e-mail: eric.homer@byu.edu. 7.—e-mail: peterh@berkeley.edu
}

The deformation of materials at cryogenic temperature is of interest for space, arctic, and fundamental science applications. In this work, a custom-built cooling system attached to a commercial picoindenter was used for in situ cryogenic microcompression testing of equal-channel angular-pressed copper with real-time electron backscatter diffraction. Stress-driven grain growth at cryogenic temperatures was observed during a series of elastic and plastic deformations. These results provide direct evidence for the previously predicted phenomenon, whereas previous ex situ examinations demonstrated coarsening after cryogenic loading when samples were not maintained at cryogenic temperatures between deformation and characterization.

\section{INTRODUCTION}

Grain growth is an important mechanism for microstructural evolution, most often observed to occur at elevated temperatures where thermal energy facilitates diffusive boundary migration. ${ }^{1,2}$ Stress has been demonstrated to be a driving force that biases grain growth under conditions where thermal energy would be insufficient, ${ }^{3}$ a phenomenon that has attracted significant interest in recent years. ${ }^{4-7}$

Zhang et al. and Brons et al. demonstrated in ex situ experiments that stress-driven coarsening may occur even at cryogenic temperatures. ${ }^{8-11}$ Zhang et al. performed ambient and cryogenic indentation of nanocrystalline copper, followed by ambienttemperature ex situ characterization with a transmission electron microscope (TEM) of both samples; interestingly, the observed coarsening was more dramatic for the sample indented at cryogenic temperatures. ${ }^{9,10}$ Brons et al. followed this work with similar ex situ indentation and characterization and demonstrated that the coarsening of different textures was correlated with the relative increase or decrease of specific grain boundary types; namely, $\langle 100\rangle$ nanotwinned $\mathrm{Cu}$ experienced an increase in $\Sigma 3$ and $\Sigma 5$ boundary content, while $\langle 111\rangle$ nanotwinned $\mathrm{Cu}$ revealed a modest decrease in $\Sigma 3$ content. co,11 $^{8,11}$

Unfortunately, the ex situ nature of those experiments leaves some question about when the coarsening occurred. It could have occurred at the cryogenic temperatures, which is supported by the decrease in the indentation load at cryogenic temperatures ${ }^{9,10}$; However, it is also possible that the cryogenic temperatures simply prevented dynamic grain coarsening and encouraged the retention of the deformation energy in the system. As a result, coarsening could have occurred once the samples returned to ambient temperature. Therefore, direct evidence of grain growth during deformation at cryogenic temperatures is still missing. The work presented herein fills this gap and provides direct proof of the phenomenon.

A custom in situ, cryogenic, picoindenter system was built for use in a scanning electron microscope (SEM). Furthermore, the system also enables orientation imaging microscopy to index electron backscatter diffraction (EBSD) patterns to characterize the microstructural evolution. The system is a 
natural evolution of instrumented indentation with load and depth sensing ${ }^{12,13}$ that has enabled significant micro- and nanoscale mechanical testing across a wide array of applications ${ }^{14,15}$ and material properties. ${ }^{16-18}$ In situ capabilities provide the ability to watch the deformation of the micro- or nanoscale specimen in real time, and also provide atmospheric control through the vacuum of the microscope. Cryogenic in situ micromechanical testing is a more recent capability, with only a handful of custom-built systems available. ${ }^{19-23}$

Through the use of in situ cryogenic experiments, it is demonstrated herein that stress-driven grain coarsening actually can occur at cryogenic temperatures. The phenomenon is demonstrated in equalchannel angular-pressed (ECAP) copper tested on the in situ system described. The in situ system allows real-time EBSD of the microcompression specimen before, during, and after testing to examine the microstructural evolution. The following sections detail the setup of the in situ system as well as the experiments, results, and a discussion of the system and results.

\section{EXPERIMENTAL PROCEDURES}

The material used in this investigation was ECAP, ultrahigh-purity, ultrafine-grained copper, which has $15 \% 50 \mathrm{~nm}$ to $200 \mathrm{~nm}$ dislocation-free grains, $37 \% 100 \mathrm{~nm}$ to $200 \mathrm{~nm}$ grains with chaotically distributed dislocations, and $48 \% 100 \mathrm{~nm}$ to $500 \mathrm{~nm}$ grains with subboundaries. ${ }^{24}$ ECAP copper was chosen for this work due to its ultrafine grain structure that (1) permits a larger number of grains in a microscale compression test ${ }^{24}$ and (2) allows for multiple grains to be present on the surface for EBSD characterization. The ECAP material originated from a 25-mm-diameter rod. The severe plastic deformation processing involved pressing a copper billet through a $90^{\circ}$ die eight times while rotating the billet $90^{\circ}$ after every other deformation pass. A full description of the processing, often described as route Bc in ECAP literature, can be found in Ref. 24 .

The microcompression specimens of ECAP copper were milled in an FEI FEG Quanta dual-beam scanning electron microscope/focused ion beam (SEM/FIB) instrument at the University of California, Berkeley. The milling was performed with the sample on a $45^{\circ}$ holder, which allows for milling perpendicular into two surfaces of the corner of the sample. The rough trenching of the pillars was completed with 7-15 nA, while final cleaning was performed with $0.5-1 \mathrm{nA}$. The final milled microcompression specimens had dimensions of $\sim 2 \mu \mathrm{m} \times$ $2 \mu \mathrm{m} \times 5 \mu \mathrm{m}$; finished microcompression specimens are shown in Fig. 1a.

The ECAP copper microcompression specimens were mounted in a modified Hysitron PI-85 system for cryogenic testing. A custom-built cryogenic cooling system was manufactured by Hummingbird Scientific for use in conjunction with the Hysitron
PI-85 picoindenter (Fig. 1b). ${ }^{23}$ The cryogenic system cools the tip and sample simultaneously to minimize thermal drift. The cooling data and system stability can be seen in Ref. 23. A flatpunch conical diamond tip with diameter of $5 \mu \mathrm{m}$ was used for the microcompression tests. It is necessary to perform these tests under vacuum and good environmental control since otherwise ice will form on the tip and specimen from humidity in the air, which has been a major hurdle to the development of cryogenic nanoindentation systems.

The microcompression specimens were mounted in the PI-85 at an angle of $35^{\circ}$ to $45^{\circ}$, as seen in Fig. 1c, so EBSD patterns could be obtained. The PI85 was then loaded into the instrument with the cooling stage on the opposite side of the Oxford EBSD detector. Software for data capture and analysis include AZtec and Channel 5, respectively.

Once the chamber had been pumped to vacuum, the whole PI-85 setup, including the ECAP copper specimens and cooling stage, was tilted $25^{\circ}$ to $35^{\circ}$. The "pre-tilt" loading of the sample plus the additional tilt in the SEM/FIB enables the face of the microcompression specimens to lie at $\sim 70^{\circ}$ with respect to the SEM beam, which is the optimal angle for EBSD measurements. Note that the specimens may not have been at exactly $70^{\circ}$, leading to some uncertainty in the absolute orientations; however, the interest here is in the relative orientations of the grains, which gives the grain boundary character, as well as the evolution of the microstructure.

Pretesting EBSD maps were taken at room temperature to verify the geometry and function of all the components prior to cooling the specimen. Subsequently the specimen was cooled to cryogenic temperature using chilled nitrogen gas flowing through a heat exchanger in liquid nitrogen. After the specimen had reached thermal equilibrium (138 K) with the cooling block, additional EBSD scans were taken at cryogenic temperature to verify the function and preexperimental condition of the system.

The microcompression specimens were tested using the PI-85 in the load-controlled mode. Since the aim of this study is to evaluate stress-driven grain coarsening at cryogenic temperature, the microcompression specimens were loaded in both the plastic and elastic regime before a final plastic deformation test; the experimental literature on stress-driven grain coarsening at cryogenic temperature is based on plastic deformation. ${ }^{8-11}$ Figure $2 \mathrm{a}$ shows the planned schedule for the loading and temperature. The schedule consists of a large plastic load, followed by several elastic loadings of $400 \mu \mathrm{N}$, and a final plastic load. The elastic loads of $400 \mu \mathrm{N}$ correspond to a stress of $\sim 82 \mathrm{MPa}$ in the microcompression specimen, which is well below the yield stress of $383 \pm 34 \mathrm{MPa}$ obtained from room-temperature microcompression testing ${ }^{24}$; a cryogenic stress-strain curve is presented in Supplementary Fig. S1. Each elastic loading was held for $300 \mathrm{~s}$ at the peak load, then a posttest EBSD scan was 

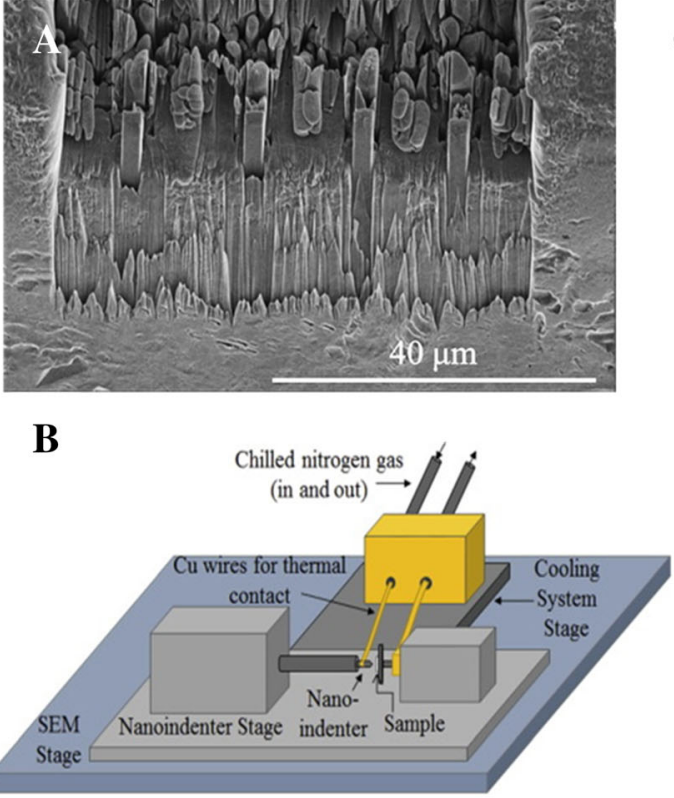

C

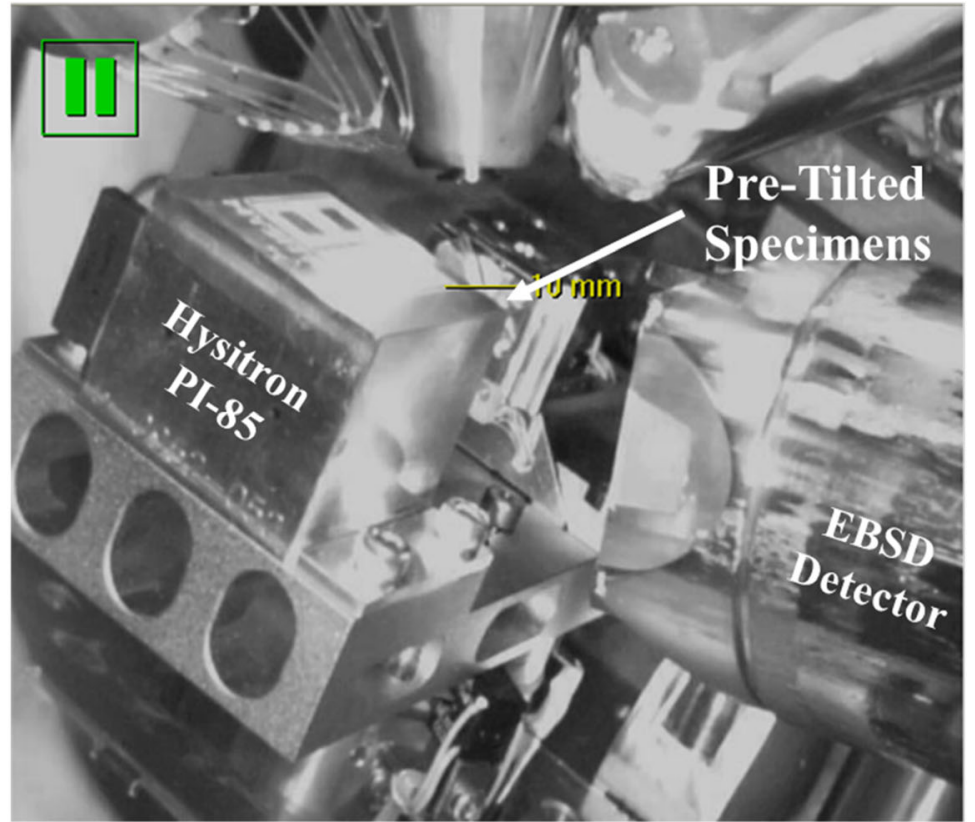

Fig. 1. (a) SEM image of ECAP copper microcompression specimens before testing. (b) Diagram illustrating cooling stage for PI-85 system, reprinted with permission from Ref. 23. (c) Infrared image of SEM/FIB chamber with EBSD camera inserted (Color figure online).

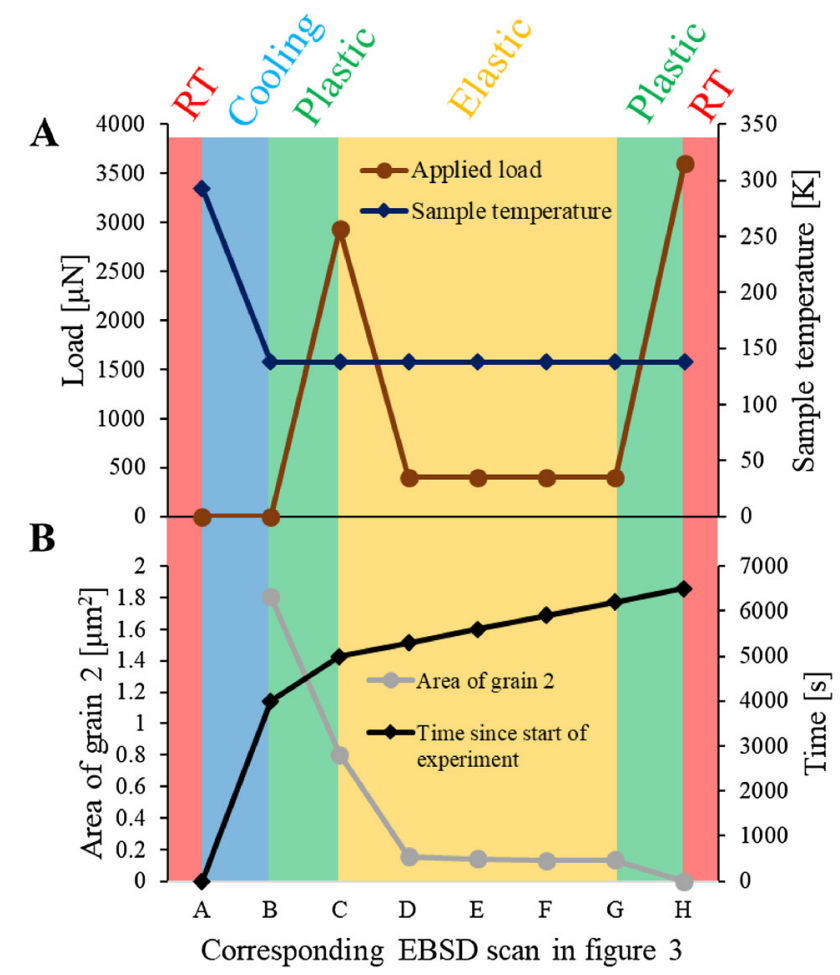

Fig. 2. Graph showing the correspondence between (a) load and temperature and (b) grain 2 area and time for various test states, including EBSD snapshots similarly identified in Fig. 3 . The microcompression specimen was cooled from RT (red) to cryogenic temperatures (blue), which takes $\sim 1 \mathrm{~h}$ for cooling and thermal stabilization. Loading included: initial plastic loading (green), where the specimen yields around $2800 \mu \mathrm{N}$; four elastic loads (yellow), each at $400 \mu \mathrm{N}$; and final plastic load (green). The specimen is then returned to RT (Color figure online). carried out. The final plastic deformation test was terminated early enough to retain a suitable surface for EBSD posttest. After all of the microcompression specimen tests, the sample and tip of the PI-85 were heated back to room temperature.

\section{RESULTS AND DISCUSSION}

Figure 2a shows the planned and executed schedule for loading and temperature. Figure 3 shows the pre- and posttest EBSD maps of a small region of the pillar corresponding to the load/temperature schedule shown in Fig. 2. The approximate pillar outline and orientation are shown in Fig. 3a. Unindexed pixels in the pillar region primarily occur at grain boundaries and from progressive deformation, both of which cause signal degradation and make accurate determination of orientation difficult.

The plastic loading causes significant changes to the microstructure, as can be seen in Fig. $3 \mathrm{~b}$ and c. The most dramatic change occurs in the brown grain, labeled as grain 2 in Fig. 3a. Figure 3b shows the change of the grain area for grain 2 , which experiences a $55 \%$ reduction in area during the plastic loading; grain areas were calculated using ImageJ software. The misorientations between the grains in Fig. 3a are presented in Table I. It appears that grain 2 is embedded inside grain 1, where the boundary between them has a misorientation of $\sim 6.4^{\circ}$, indicating that it is a low-angle grain boundary. Note that a threshold of $4^{\circ}$ is used to determine when a change in orientation is sufficient to be identified as a grain boundary in the orientation maps. 


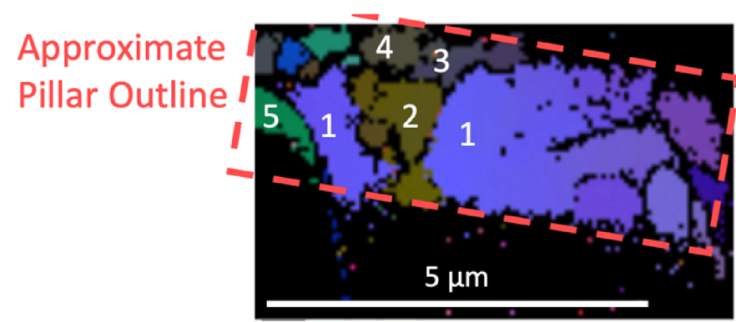

A Room Temperature

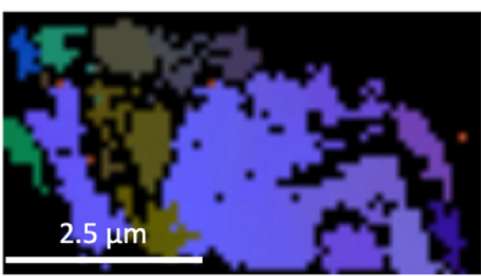

B Cryo before load

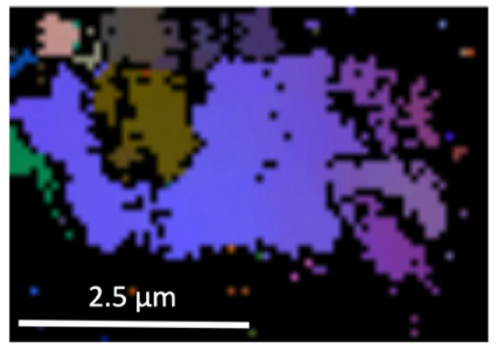

C After $1^{\text {st }}$ load (plastic)

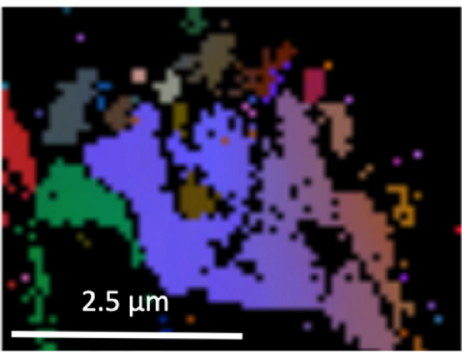

F After $4^{\text {th }}$ load (elastic)

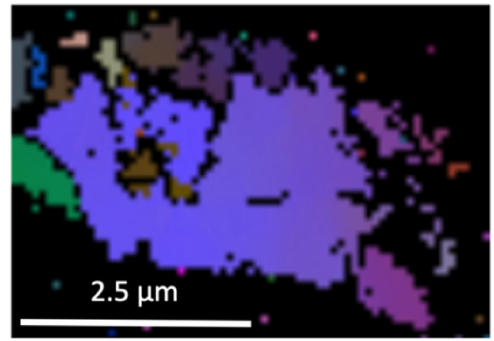

D After $2^{\text {nd }}$ load (elastic)

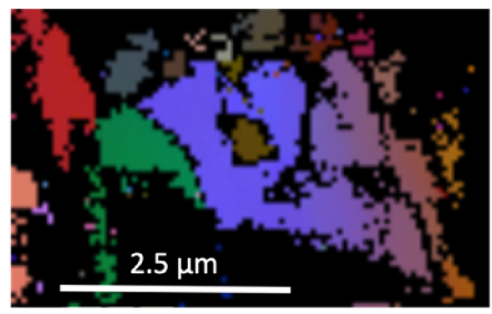

G After $5^{\text {th }}$ load (elastic)

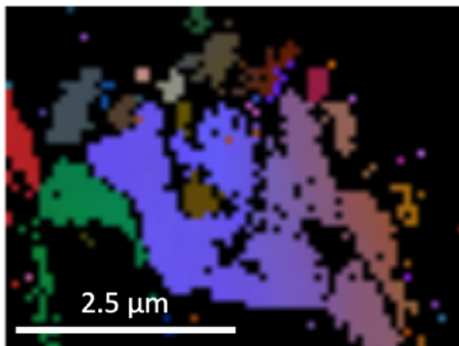

E After $3^{\text {rd }}$ load (elastic)

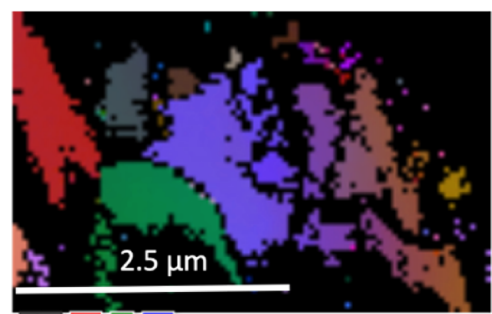

H After $6^{\text {th }}$ load (plastic)

Fig. 3. (a-h) Grain maps at each step illustrated in Fig. 2. Grain 2 contracts significantly between (b) and (c), between (c) and (d), and again between $(\mathrm{g})$ and $(\mathrm{h})$. The scale bar change between room and cryogenic temperature (a and b) results from the need to recenter the specimen after cooling-caused shrinkage (Color figure online).

Table I. Boundary information for all grains labeled in Fig. 3a, including misorientation angle, axis of rotation, and offset angle of actual axis of rotation from the integer axis of rotation listed

\begin{tabular}{|c|c|c|c|}
\hline $\begin{array}{l}\text { Grain } \\
\text { boundary }\end{array}$ & Misorientation & $\begin{array}{c}\text { Axis of } \\
\text { rotation }\end{array}$ & Offset \\
\hline $1-2$ & $6.4^{\circ}$ & {$[23 \overline{1}]$} & $3.42^{\circ}$ \\
\hline $1-3$ & $22.5^{\circ}$ & {$[340]$} & $3.61^{\circ}$ \\
\hline $2-3$ & $12.5^{\circ}$ & {$[\overline{3} \overline{4} \overline{1}]$} & $3.12^{\circ}$ \\
\hline $2-4$ & $10^{\circ}$ & {$[\overline{1} \overline{2} 0]$} & $5.57^{\circ}$ \\
\hline $3-4$ & $6.7^{\circ}$ & {$[320]$} & $4.66^{\circ}$ \\
\hline $1-5$ & $56.6^{\circ}(\Sigma 3)$ & {$[\overline{1} 11]$} & $3.13^{\circ}$ \\
\hline
\end{tabular}

The results of the series of elastic loads and holds can be seen in Fig. 3d, e, $f$ and $g$. The first elastic load causes a significant reduction for grain 2 , resulting in a further $80.7 \%$ reduction; this indicates that both elastic and plastic loading is sufficient to cause the microstructural coarsening. However, the remaining elastic loads appear to cause very little additional change, as indicated by the snapshots in Fig. 3 and the grain area in Fig. 2b. The final plastic loading causes grain 2 to almost completely disappear; a small patch of grain 2 remains at the top of Fig. $3 \mathrm{~h}$, but it is not included in the area calculations in Fig. 2 b.

The in situ cryogenic microcompression tests provide clear evidence of microstructural coarsening. While there are a variety of factors that could, or would have been expected to play a role here, we discuss a few of the most relevant.

It is interesting to note that the grain that shrinks the most, grain 2, is surrounded on most sides by a low-angle grain boundary. The presence of this low-angle grain boundary is confirmed in the local misorientation map in Fig. 4a. Furthermore, Fig. 4b demonstrates that the region previously occupied by grain 2 has low magnitudes of local misorientation changes. This is consistent with a recrystallizing grain consuming a deformed region, leaving behind a region with little deformation. It should be noted, however, that unlike 

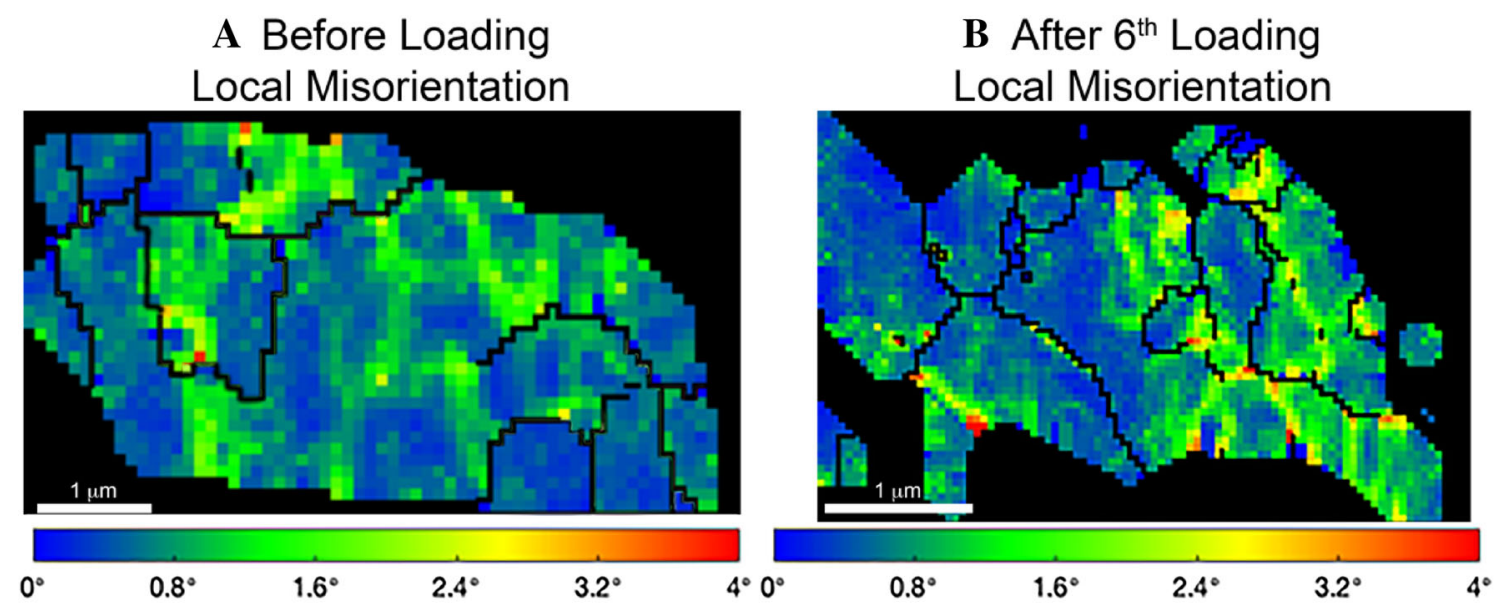

Fig. 4. Local misorientation changes (a) before and (b) after applied load demonstrates changes caused by the boundary motion (Color figure online).

recrystallization, the grain that grows is not free from deformation, except in the swept region, since the initial material is heavily deformed ECAP copper.

This observation begs the question of whether the boundary migration is driven by the inherent mobility of the boundary, the deformation structure, or disparate grain sizes.

Simulations of grain boundary migration suggest that specific boundaries are likely to be mobile at cryogenic temperatures, such as $\Sigma 3, \Sigma 7, \Sigma 9$, and $\Sigma 11$ coincident site lattice (CSL) boundaries. ${ }^{25-31}$ Unfortunately, simulations have not examined the cryogenic mobility of low-angle grain boundaries, such as the one that consumes grain 2. However, Brons et al. noted that low-angle boundaries can experience significant evolution in cryogenic indentation, depending upon the location relative to the indentation. ${ }^{8}$ Furthermore, Brons et al. also find that some of these same CSL boundaries are involved with the cryogenic coarsening. ${ }^{6}$ Thus, there is some indication that specific boundaries might have inherent mobilities that facilitate cryogenic coarsening.

On the other hand, room-temperature in situ, $\mathrm{x}$ ray Laue diffraction microscopy examining the three-dimensional (3D) migration of a recrystallizing boundary suggest a different conclusion. ${ }^{32}$ In that work, certain facets of a recrystallizing boundary migrated, while other facets did not. Together with accompanying simulations of the same boundaries, except without a deformed structure, ${ }^{33}$ the results indicate that the nature of the deformation microstructure plays a greater role in the migration of the recrystallizing boundary than do the inherent mobilities of the boundaries in question.

This is possibly supported by the fact that the one $\Sigma 3$ CSL boundary in the present sample did not migrate. Simulations indicate that most $\Sigma 3$ boundary plane orientations, excluding the coherent twin plane, are expected to be mobile at cryogenic temperatures. ${ }^{27}$ Without the full five-dimensional (5D) characterization (misorientation and boundary plane orientation) of the boundaries, one cannot definitively say whether the $\Sigma 3$ CSL boundary in the present sample would have been expected to be mobile.

This could also be the result of abnormal grain growth. The grains in question have grain sizes much larger than the original grain size of the sample (grain 2 is $\sim 1.5 \mu \mathrm{m}$ wide while grain 1 is $\sim 5 \mu \mathrm{m}$ wide, both being much larger than the $\sim 200 \mathrm{~nm}$ grain size). Perhaps this abnormally large grain will consume its surrounding neighbors regardless of their nature, as routinely observed in abnormal grain growth. On the other hand, grain 1 appears to preferentially consume grain 2 , and not all other surrounding grains, though this is difficult to see with the collected data.

Thus, while the present work shows clear evidence for stress-driven microstructural coarsening at cryogenic temperatures, the underlying cause of the coarsening requires further investigation. Furthermore, the grain size and deformed nature of the present sample ${ }^{24}$ differ from the undeformed and fine structures of the previous studies, ${ }^{8-11}$ such that the mechanism and/or driving force for the coarsening could be different.

It should also be noted that, while the in situ SEM testing makes this observation possible and removes some of the challenges of ex situ testing, it is not without challenges. In particular, there could be gallium contamination of the microcompression sample from the manufacturing. Gallium implanted during milling could inhibit or prevent the hypothesized cryogenic migration behaviors. Also, it may be possible that the loading conditions in this test were insufficient to induce the hypothesized migration behaviors in the other grain boundaries. 


\section{CONCLUSION}

In situ SEM cryogenic testing of samples on the microscale with real-time microstructure characterization revealed cryogenic stress-driven grain coarsening, providing direct proof of this previously hypothesized phenomenon ${ }^{8-11}$ for the first time. The setup utilized here allows the microstructure to be characterized following several loading cycles, ensuring that all changes that occur do so at cryogenic temperatures, and not during any subsequent processing for posttest characterization. The major microstructural coarsening involves a lowangle grain boundary. The cause of the boundary migration could be dominated by the inherent mobility of the boundary, and it could be dominated by the underlying deformation microstructure. This work proves that grain growth can occur at cryostatic temperatures in ultrafine-grained and nanograined materials.

\section{ACKNOWLEDGEMENTS}

The authors acknowledge Daniel Kiener from the Erich Schmidt Institute in Austria and Brad Boyce from Sandia National Laboratory for encouraging discussion on this topic.

\section{FUNDING}

Research supported as part of FUTURE (Fundamental Understanding of Transport Under Reactor Extremes), an Energy Frontier Research Center funded by the US Department of Energy (DOE), Office of Science, Basic Energy Sciences (BES) (neutron scattering studies) and by the US Department of Energy (DOE), Office of Science, Basic Energy Sciences (BES) under Award \#DESC0016441.

\section{ELECTRONIC SUPPLEMENTARY MATERIAL}

The online version of this article (https://doi.org/ 10.1007/s11837-020-04075-x) contains supplementary material, which is available to authorized users.

\section{REFERENCES}

1. A.P. Sutton and R.W. Balluffi, Interfaces in Crystalline Materials (Oxford: Oxford University Press, 1995).

2. G. Gottstein and L.S. Shvindlerman, Grain Boundary Migration in Metals: Thermodynamics, Kinetics, Applications, 2nd ed. (London: Taylor \& Francis Group, 2010).
3. D.W. Bainbridge, H.L. Choh, and E.H. Edwards, Acta Met. Mater. 2, 322 (1954). https://doi.org/10.1016/0001-6160(54)9 0175-3.

4. J.F. Panzarino, Z. Pan, and T.J. Rupert, Acta Mater. 120, 1 (2016).

5. T.J. Rupert, D.S. Gianola, Y. Gan, and K.J. Hemker, Science 326, 1686 (2009).

6. C.V. Thompson and R. Carel, J. Mech. Phys. Solids 44, 657 (1996).

7. J.A. Sharon, P.-C. Su, F.B. Prinz, and K.J Hemker, Scr. Mater. 64, 25 (2011).

8. J.G. Borns, J.A. Hardwick, H.A. Padilla II, K. Hattar, G.B. Thompson, and B.L. Boyce, Mater. Sci. Eng. A 592, 182 (2014).

9. K. Zhang, J.R. Weertman, and J.A. Eastman, Appl. Phys. Lett. 87, 061921 (2005).

10. K. Zhang, J.R. Weertman, and J.A. Eastman, Appl. Phys. Lett. 85, 5197 (2004).

11. J.G. Brons, H.A. Padilla II, G.B. Thompson, and B.L. Boyce, Scr. Mater. 68, 781 (2013).

12. J.L. Loubet, J.M. Georges, O. Marchesini, and G. Meille, J. Tribol. 106, 43 (1984).

13. D. Newey, M.A. Wilkens, and H.M. Pollock, J. Phys. 15, 119 (1982).

14. X. Li and B. Bhushn, Mater. Character. 48, 11 (2002).

15. S.J. Bull, J. Phys. D: Appl. Phys. 38, 393 (2005).

16. M.D. Uchic, D.M. Dimiduk, J.N. Florando, and W.D. Nix, Science 305, 986 (2004).

17. P. Hosemann, Scr. Mater. 143, 161 (2018).

18. M.D. Abad, S. Parker, D. Frazer, M.R. de Figueiredo, A. Lupinacci, K. Kikuchi, and P. Hosemann, Oxid. Met. 84, 211 (2015).

19. B.D. Snartland, A.B. Hagen, and C. Thaulow, Eng. Fract. Mech. 175, 312 (2017).

20. S.-W. Lee, Y.T. Cheng, I. Ryu, and J.R. Greer, Sci. China Technol. Sci. 57, 652 (2014).

21. A.B. Hagen and C. Thaulow, Mater. Sci. Eng. A 678, 355 (2016).

22. S.-W. Lee, L. Meza, and J.R. Greer, Appl. Phys. Lett. 103, 101906 (2013).

23. A. Lupinacci, J. Kacher, A. Eilenberg, A.A. Shapiro, P. Hosemann, and A.M. Minor, Acta Mater. 78, 56 (2014).

24. C. Howard, D. Frazer, A. Lupinacci, S. Parker, R.Z. Valiev, C. Shin, B.W. Choi, and P. Hosemann, Mater. Sci. Eng. A 649, 104 (2016).

25. J.L. Bair and E.R. Homer, Acta Mater. 162, 10 (2019).

26. J.L. Priedeman, D.L. Olmsted, and E.R. Homer, Acta Mater. 131, 553 (2017).

27. J. Humberson, and E.A. Holm, https://arxiv.org/abs/1704.0 3088.

28. D.L. Olmsted, E.A. Holm, and S.M. Foiles, Acta Mater. 573, 704 (2009).

29. E.R. Homer, E.A. Holm, S.M. Foiles, and D.L. Olmsted, JOM 66, 114 (2014).

30. E.R. Homer, S. Patala, and J.L. Priedeman, Sci. Rep. 515, 15476 (2015).

31. J. Humberson and E.A. Holm, Scr. Mater. 130, 1 (2017).

32. Y.B. Zhang, J.D. Budai, J.Z. Tischler, W. Liu, R. Xu, E.R. Homer, A. Godfrey, and D. Juul Jensen, Sci. Rep. 7, 1 (2017).

33. E.R. Homer, 36th Riso International Symposium on Materials Science, IOP Publishing, Roskilde (2015), p. 012006.

Publisher's Note Springer Nature remains neutral with regard to jurisdictional claims in published maps and institutional affiliations. 